\title{
Switching Regimes in Economics: The Contraction Mapping and the $\omega$-Limit Set
}

\author{
Pascal Stiefenhofer ${ }^{1,2}$, Peter Giesl ${ }^{1}$ \\ ${ }^{1}$ Department of Mathematics, University of Sussex, Falmer, East Sussex, UK \\ ${ }^{2}$ Brighton Business School, University of Brighton, Brighton, UK \\ Email: p.stiefenhofer@sussex.ac.uk,p.stiefenhofer@brighton.ac.uk,P.A.Giesl@sussex.ac.uk
}

How to cite this paper: Stiefenhofer, P. and Giesl, P. (2019) Switching Regimes in Economics: The Contraction Mapping and the $\omega$-Limit Set. Applied Mathematics, 10, 513-520.

https://doi.org/10.4236/am.2019.107035

Received: May 14, 2019

Accepted: June 30, 2019

Published: July 3, 2019

Copyright ( 2019 by author(s) and Scientific Research Publishing Inc. This work is licensed under the Creative Commons Attribution International License (CC BY 4.0).

http://creativecommons.org/licenses/by/4.0/ (c) (i) Open Access

\begin{abstract}
This paper considers a dynamical system defined by a set of ordinary autonomous differential equations with discontinuous right-hand side. Such systems typically appear in economic modelling where there are two or more regimes with a switching between them. Switching between regimes may be a consequence of market forces or deliberately forced in form of policy implementation. Stiefenhofer and Giesl [1] introduce such a model. The purpose of this paper is to show that a metric function defined between two adjacent trajectories contracts in forward time leading to exponentially asymptotically stability of (non)smooth periodic orbits. Hence, we define a local contraction function and distribute it over the smooth and nonsmooth parts of the periodic orbits. The paper shows exponentially asymptotical stability of a periodic orbit using a contraction property of the distance function between two adjacent nonsmooth trajectories over the entire periodic orbit. Moreover it is shown that the $\omega$-limit set of the (non)smooth periodic orbit for two adjacent initial conditions is the same.
\end{abstract}

\section{Keywords}

Non-Smooth Periodic Orbit, Differential Equation, Contraction Mapping, Economic Regimes, Non-Smooth Dynamical System

\section{Introduction}

Economic systems may not always satisfy the usual smoothness condition everywhere. In particular, a discontinuity in an economic system may occur due to a change in economic regime or policy implementation. In this paper, we consider an economic system defined by a planar ordinary differential equation with discontinuous right-hand side. Similar dynamical systems are considered in 
various economic models [2] [3] [4] [5] [6]. For such models, there exists a well developed existence and uniqueness theory [7]. However, little is known about stability results of non-smooth periodic orbits. Moreover, such results depend on the explicit calculation of the periodic orbit and employ a global stability theory based on Poincaré's map. Since such explicit calculations may not always be possible, we want to establish existence and exponentially asymptotical stability of a nonsmooth periodic orbit without its calculation. The advantage of such a local stability theory would allow economists to derive analytic results for the purpose of economic policy analysis. The theory developed in Stiefenhofer and Giesl [1] allows us to do so. In this paper we study the distance function between two adjacent trajectories and show it's contraction property in forward time and calculate its $\omega$-limit set. Section two discusses the dynamical system with a switching regime and recalls the theorem introduced in Stiefenhofer and Giesl [1]. Section three states the main results and provides the proofs. Section four is a conclusion.

\section{The Model}

We consider a differential equation

$$
\dot{x}=f(x),
$$

where $f$ is a discontinuous function at $x_{2}=0$ and $x \in \mathbb{R}^{2}$ such that for $f:=f^{ \pm}$we have

$$
\dot{x}=f^{ \pm}(x)= \begin{cases}f^{+}(x) & \text { if } x_{2}>0 \\ f^{-}(x) & \text { if } x_{2}<0\end{cases}
$$

This dynamical system is introduced in Stiefenhofer and Giesl [8]. On the right-hand side, we provide a condition for switching between economic regimes $f^{ \pm}$. For simplicity, we consider only two regimes and an exogenously given switching condition between them. A stability theory for this dynamical system is provided by the following theorem.

Theorem 1 (Stiefenhofer and Giesl [1], Theorem 2 p. 11). Let $\varnothing \neq K \subset \mathbb{R}^{2}$ be a compact, connected and positively invariant set with $f^{ \pm}(x) \neq 0$ for all $x \in K^{ \pm}$. Moreover, assume that $W^{ \pm}: \mathbb{X}^{ \pm} \rightarrow \mathbb{R}^{2}$ are continuous functions and let the orbital derivatives $\left(W^{ \pm}\right)^{\prime}$ exist and be continuous functions in $\mathbb{X}^{ \pm}$and continuously extendable up to $\mathbb{X}_{0}^{ \pm}$. Let following conditions hold:

1) $L_{W^{ \pm}(x)}:=\max _{\left\|v^{ \pm}\right\|=\mathrm{e}^{-W^{ \pm}(x)}, v^{ \pm} \perp f^{ \pm}(x)} L_{W^{ \pm}}\left(x, v^{ \pm}\right) \leq-v<0$

$$
L_{W^{ \pm}}\left(x, v^{ \pm}\right):=\mathrm{e}^{2 W^{ \pm}(x)}\left\{\left(v^{ \pm}\right)^{\mathrm{T}}\left[D f^{ \pm}(x)\right] v^{ \pm}+\left\langle\nabla W^{ \pm}(x), f^{ \pm}(x)\right\rangle\left\|v^{ \pm}\right\|^{2}\right\}
$$

for all $x \in K^{ \pm}$.

2) $\frac{f_{2}^{\mp}\left(x_{1}, 0\right)}{f_{2}^{ \pm}\left(x_{1}, 0\right)} \cdot \frac{\sqrt{\left(f_{1}^{ \pm}\left(x_{1}, 0\right)\right)^{2}+\left(f_{2}^{ \pm}\left(x_{1}, 0\right)\right)^{2}}}{\sqrt{\left(f_{1}^{\mp}\left(x_{1}, 0\right)\right)^{2}+\left(f_{2}^{\mp}\left(x_{1}, 0\right)\right)^{2}}} \mathrm{e}^{W^{\mp}\left(x_{1}, 0\right)-W^{ \pm}\left(x_{1}, 0\right)}<1$

for all $x \in K^{0}$ with $f_{2}^{ \pm}\left(x_{1}, 0\right)<0, \quad f_{2}^{\mp}\left(x_{1}, 0\right)<0$. 
Then there is one and only one periodic orbit $\Omega \subset K$. Moreover, $\Omega$ is exponentially asymptotic stable with the real part of the Floquet exponent less or equal $-v$ except the trivial one and for its basin of attraction the inclusion $K \subset A(\Omega)$ holds.

Stiefenhofer and Giesl [1] derive the conditions 1 - 2 in theorem 1. Condition 1 states that two smooth trajectories contract if the weighted Lyapunov function $L_{W^{ \pm}}$is negative. This condition requires that the real part of the Floquet exponent be negative. While this condition does not depend on the periodic orbit itself, however, it requires to find a function $W^{ \pm}(x)$. Condition 2 states a contraction property for the discontinuity points of the dynamical system, where the system switches. This condition depends on the vector field $f^{ \pm}$and some function $W^{ \pm}$, and is hence independent of the periodic orbit itself. We now investigate the contraction property of the metric function between adjacent solutions, and calculate the $\omega$-limit set of the periodic orbit. The details of how to derive these conditions are given in [8]. In principle, however, our method is a generalization of Borg [9], which introduces the concept of a contraction mapping between adjacent trajectories in the following way:

We want to show that $L(x)<0$ is a sufficient condition for two adjacent trajectories to move towards each other. For example, consider the points $x \in \mathbb{R}^{n}$ and $x+\delta v \in \mathbb{R}^{n}$ in the phase space. Let $\delta>0, v \perp f(x)$, and $\|v\|=1$. Then in order for two adjacent trajectories through the points $x$ and $x+\delta v$ to move towards each other it must hold that

$$
\begin{gathered}
0>\langle f(x+\delta v), v\rangle \\
\approx\langle f(x)+\delta D f(x) v, v\rangle \\
=\delta\langle D f(x) v, v\rangle \text { since } v \perp f(x) .
\end{gathered}
$$

where

$$
L(x, v):=\langle D f(x) v, v\rangle .
$$

Hence, if $L(x)<0$ then locally, two adjacent trajectories move towards each other. See Figure 1. Borg provides the following theorem under slightly different assumptions:

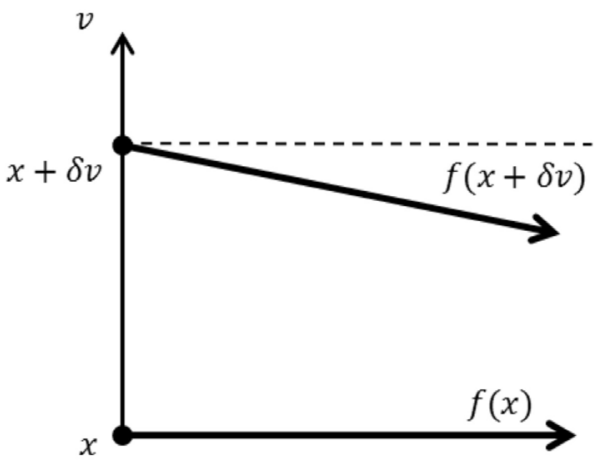

Figure 1. Borg's criterion [9]. 
Theorem 2 (Version of Borg [9]) Let $\varnothing \neq K \subset \mathbb{R}^{n}$ be a compact, connected and positively invariant set which contains no equilibrium. Let $L(x)<0$ hold for all $x \in K$ with

$$
L(x):=\max _{\|v\|=1, v \perp f(x)}(x, v)
$$

where

$$
L(x, v):=\langle D f(x) v, v\rangle .
$$

Then there exists one and only one periodic orbit $\Omega \subset K . \Omega$ is exponentially asymptotically stable and its basin of attraction $A(\Omega)$ contains $K$.

\section{Results}

We now consider the time interval $t \in\left(t_{j-1}^{+}, t_{j}^{+}\right)$in Figure 2 and show that the distance between two adjacent solutions decreases. We also show that for two nearby points $x$ and $x+\eta$ in $K$ that the $\omega$-limit set is the same.

We define a time-dependent distance function $A^{+}: \mathbb{R} \rightarrow \mathbb{R}_{0}^{+}$between two adjacent points $x$ and $x+\eta$ by

$$
A^{+}(t):=\sqrt{\left(\left(S_{\mathcal{T}_{x}^{x+\eta}(t)}^{+}(x+\eta)-S_{t}^{+} x\right)^{\mathrm{T}} \mathrm{e}^{2 W^{+}\left(S_{t}^{+} x\right)}\left(S_{\mathcal{T}_{x}^{x+\eta}(t)}^{+}(x+\eta)-S_{t}^{+} x\right)\right)},
$$

where $S_{\mathcal{T}_{x}^{x+\eta}}(t)(x+\eta), S_{t}^{+} x$ are two adjacent (non)smooth trajectories, and $\mathcal{T}_{x}$ is a monotone increasing map for the time structure presented in Figure 2.

Theorem 3. Let the assumptions of theorem 1 hold. Then there are constants $\delta>0$ and $C \geq 1$ such that for all $x \in K$ and for all $\eta \in \mathbb{R}^{2}$ with $\|\eta\| \leq \delta / 2$

$$
A(t) \leq A\left(t_{j-1}^{+}\right) \mathrm{e}^{-\mu t} \text { for all } t \geq 0 .
$$

Moreover, we have

$$
\omega(x)=\omega(x+\eta) .
$$

Proof.

We now show the contraction property of the distance function.

- We show that $v$ defined over a smooth time interval is strictly larger than $\mu$ defined over the same time interval including the subsequent time interval.

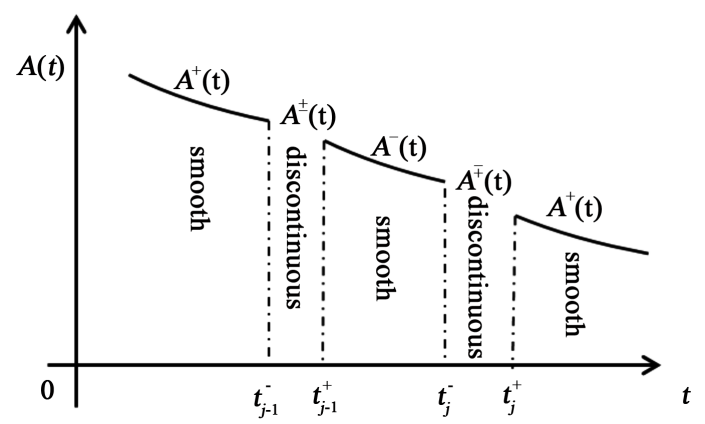

Figure 2. Time structure $t \in \mathbb{R}$. 
- We show that the distance function is decreasing for all positive time.

By equations (2.17) and (2.30) in Stiefenhofer [8] we have

$$
\begin{gathered}
A(t) \leq \mathrm{e}^{-\mu t} A^{+}\left(t_{j-1}^{-}\right) \text {for all } t \in\left[t_{j-1}^{-}, t_{j-1}^{+}\right] \\
A(t) \leq \mathrm{e}^{-\mu t} A^{+}\left(t_{j}^{-}\right) \text {for all } t \in\left[t_{j}^{-}, t_{j}^{+}\right]
\end{gathered}
$$

See time structure notation of the graph of $A(t)$ in Figure 2. Equations (12) and (13) show the contraction rate $\mu$ over each jumping interval in $+/-$ and in $-/+$ direction. We now state similar equations for the smooth intervals with contraction rate $v$. We have

$$
\begin{aligned}
& A(t) \leq \mathrm{e}^{-v t} A\left(t_{j-1}^{+}\right)^{-} \text {for all } t \in\left(t_{j-1}^{+}, t_{j}^{-}\right) \\
& A(t) \leq \mathrm{e}^{-v t} A\left(t_{j}^{+}\right) \text {for all } t \in\left(t_{j}^{+}, t_{j+1}^{-}\right)^{-} .
\end{aligned}
$$

We consider the time interval $\left[t_{1}^{-}, t_{1}^{+}\right] \cup\left(t_{1}^{+}, t_{2}^{-}\right)$. Hence by equation (12) and equation (14) we obtain

$$
\begin{gathered}
\mathrm{e}^{-v\left(t_{2}^{-}-t_{1}^{+}\right)} A^{+}\left(t_{1}^{+}\right) \leq \mathrm{e}^{-\mu\left(t_{2}^{+}-t_{1}^{+}\right)} A^{+}\left(t_{1}^{+}\right) \\
v\left(t_{2}^{-}-t_{1}^{+}\right) \leq \mu\left(t_{2}^{+}-t_{1}^{+}\right) \\
\mu \leq v\left(\frac{t_{2}^{-}-t_{1}^{+}}{t_{2}^{+}-t_{1}^{+}}\right) .
\end{gathered}
$$

We define

$$
\begin{gathered}
S:=\left(t_{j}^{-}-t_{j-1}^{+}\right) \geq c_{2} \text { for } j=1,2,3, \cdots \\
J:=\left(t_{j}^{+}-t_{j}^{-}\right) \leq c_{1} \text { for } j=1,2,3, \cdots
\end{gathered}
$$

where constants $c_{1}, c_{2}>0$ are defined by

$$
c_{1}:=\delta>0
$$

For the constant $c_{2}$ we consider $d:=K \cap\left\{x_{2}=0\right\}$. From

$$
\max _{x \in K}\left|f_{1}(x)\right|=s
$$

and

$$
t \cdot s=d
$$

we obtain by $d \leq \int_{0}^{t} f_{1}(x(\tau)) \mathrm{d} \tau$

$$
c_{2}:=\frac{d}{\max _{x \in K}\left|f_{1}\right|} \leq t .
$$

Equation (16) with bounds (17) and (18) and extension of time interval $t_{2}^{+}-t_{1}^{+}=\left(t_{2}^{-}-t_{1}^{+}\right)+\left(t_{2}^{+}-t_{2}^{-}\right)$yields

$$
\mu=v\left(\frac{t_{2}^{-}-t_{1}^{+}}{\left(t_{2}^{+}-t_{1}^{+}\right)\left(t_{2}^{+}-t_{2}^{-}\right)}\right) \leq v\left(\frac{c_{2}}{c_{2}+c_{1}}\right)=v\left(\frac{1}{1+\frac{c_{1}}{c_{2}}}\right)
$$


Since $c_{1}=\delta$ we can choose $\delta$ small enough so that $\mu$ gets as close to $v$ as we wish. From

$$
\begin{aligned}
& A\left(t_{1}^{+}+t\right) \leq \mathrm{e}^{-\mu t} A\left(t_{1}^{+}\right) \text {for all } t \in\left\{\left(t_{1}^{+}, t_{2}^{-}-\right) \cup\left[t_{2}^{-}-t_{2}^{+}\right]\right\} \\
& A\left(t_{1}^{+}+t\right) \leq \mathrm{e}^{-\mu t} A\left(t_{1}^{+}\right) \text {for all } t \in\left\{\left(t_{2}^{+}, t_{3}^{-}-\right) \cup\left[t_{3}^{-}-t_{3}^{+}\right]\right\}
\end{aligned}
$$

we have

$$
\begin{aligned}
& A\left(t_{1}^{+}+\tau\right) \leq \mathrm{e}^{-\mu \tau} A\left(t_{1}^{+}\right) \\
& \text {for all } \tau \in\left\{\left(t_{1}^{+}, t_{2}^{-}-\right) \cup\left[t_{2}^{-}-t_{2}^{+}\right] \cup\left(t_{2}^{+}, t_{3}^{-}-\right) \cup\left[t_{3}^{-}-t_{3}^{+}\right]\right\}
\end{aligned}
$$

which generalizes to $\tau \geq 0$, by

$$
\begin{aligned}
& A\left(t_{1}^{+}+\tau\right) \leq \mathrm{e}^{-\mu \tau} A\left(t_{1}^{+}\right) \\
& \text {for all } \tau \in\left\{\left(t_{j-1}^{+}, t_{j}^{-}-\right) \cup\left[t_{j}^{-}-t_{j}^{+}\right] \cup\left(t_{j}^{+}, t_{j+1}^{-}-\right) \cup\left[t_{j+1}^{-}-t_{3}^{+}\right]\right\}
\end{aligned}
$$

This shows (10). It remains to show (11).

Now, we show that all points $x+\eta$ with $\eta \in \mathbb{R}^{2}, \eta \perp f(x)$, and $\|\eta\| \leq \delta / 2$ have the same $\omega$-limit set as the point $x$. We first show the inclusion $\omega(x) \subset \omega(x+\eta)$. Assume there is a $w \in \omega(x)$. Then we have a strictly increasing sequence $t_{i} \rightarrow \infty$ satisfying $\left\|w-S_{t_{i}} x\right\| \rightarrow 0$ as $i \rightarrow \infty$. Because of condition (10) of proposition 3 and the properties of $\mathcal{T}$ in Figure 2 there is a sequence $\mathcal{T}\left(t_{i}\right)$ that satisfies

$$
\mathcal{T}\left(t_{i}\right) \rightarrow \infty \text { as } i \rightarrow \infty
$$

and

$$
A^{-}\left(t_{i}\right) \leq A^{+}\left(\mathcal{T}\left(t_{i}\right)\right) \mathrm{e}^{-\mu t_{i}} \text { as } i \rightarrow \infty .
$$

This proves that $S_{\mathcal{T ( t _ { i } )}}(x+\eta) \rightarrow w$ and $w \in \omega(x+\eta)$.

We now show that the inclusion $\omega(x+\eta) \subset \omega(x)$. Assume there is a $w \in \omega(x+\eta)$. Then we have a strictly increasing sequence $\theta_{i} \rightarrow \infty$ satisfying $\left\|w-S_{t_{i}} x\right\| \rightarrow 0$ as $i \rightarrow \infty$. Because of condition (10) of proposition 3 and properties of $\mathcal{T}$ in Figure 2 there is a sequence $\mathcal{T}^{-1}\left(\theta_{i}\right)$ that satisfies $\mathcal{T}^{-1}\left(\theta_{i}\right) \rightarrow \infty \quad$ as $i \rightarrow \infty$. This proves that $S_{\mathcal{T}\left(t_{i}\right)}(x+\eta) \rightarrow w$ and $w \in \omega(x+\eta)$.

This concludes the proof of proposition 3.

Proposition 4. Let the assumptions of theorem 1 be satisfied. Then for all $x, y \in K$

$$
\varnothing \neq \omega(x)=\omega(y)=: \Omega
$$

Proof. Let $x_{0} \in \Omega \backslash K^{0}$. Since for all $t \geq 0$ we have $S_{t} x_{0} \subset K$, which is a compact set, hence

$$
\varnothing \neq \omega\left(x_{0}\right)=: \Omega \subset K .
$$

Now, pick an arbitrary point $x_{0} \in \Omega \backslash K^{0}$. By proposition 3 we have $\omega(x)-\omega(y)$ for all $y$ in a neighbourhood of $x$. Hence

$$
K_{1}:=\left\{x \in K: \omega(x)=\omega\left(y_{0}\right)\right\}
$$




$$
K_{2}:=\left\{x \in K: \omega(x) \neq \omega\left(y_{0}\right)\right\}
$$

are open sets. Since $K=K_{1} \dot{U}_{2}$ and $p_{0} \in K_{1}$ with $K$ connected, it must be that $K_{2}$ is empty and $K_{1}=K$.

\section{Conclusion}

Differential equations are ubiquitous in economics. Economic regimes, where there is a switching between them, fit particularly well within the framework of differential equations with discontinuous right-hand side, where the discontinuity represents the switching condition. In this paper, we assume an exogenous switching condition. However, this can without loss of generality be generalized by modelling the explicit economic context. The novelty of the stability theory discussed in this paper is that it is independent of the explicit solution of the system. This is a major advantage of our theory. However, it requires defining a weight function $W$, which may not always be easy. In particular, the paper shows that a distance function between two adjacent trajectories contracts in forward time over both, smooth and nonsmooth parts of the periodic orbit, where the dynamical system is discontinuous. It also shows that for two adjacent initial points the $\omega$-limit set of nonsmooth period orbits is the same. Stiefenhofer and Giesl provide an example of the theory discussed in this paper [10] and compare it to global stability theory [11]. Further research should investigate the full basin of attraction of this model. Such a result would allow economists to fully characterize the set of initial conditions of exponentially asymptotically stable periodic orbits and to hence perform effective policy analysis.

\section{Acknowledgements}

We thank the Editor and the referee for their comments. EPSRC Research Grant (Engineering and Physical Science Research Council, 2011-2016), 1091684, Stability in Nonsmooth Systems with Applications to Biomechanics. This support is greatly appreciated.

\section{Conflicts of Interest}

The authors declare no conflicts of interest regarding the publication of this paper.

\section{References}

[1] Stiefenhofer, P. (2016) Stability Analysis of Non-Smooth Dynamical Systems with an Application to Biomechanics. PhD Thesis, University of Sussex, School of Mathematics and Physical Sciences, Brighton.

[2] Mallivaud, F. (1977) The Theory of Unemployment Reconsidered. Basil Blackwell, Oxford.

[3] Henry, C. (1972) Differential Equations with Discontinuous Right-Hand Side for Planning Procedure. Journal of Economic Theory, 4, 545-551. 
https://doi.org/10.1016/0022-0531(72)90138-X

[4] Benassy, J.P. (1978) A Neo-Keynesian Model of Price and Quantity Determination in Disequilibrium. In: Schwödiauer, G., Ed., Equilibrium and Disequilibrium in Economic Theory, Reidel, Dordrecht, 511-544. https://doi.org/10.1007/978-94-010-1155-6 27

[5] Ito, T. (1979) A Filippov Solution of a System of Differential Equations with Discontinuous Right-Hand Side. Economic Letters, 4, 349-354. https://doi.org/10.1016/0165-1765(79)90183-6

[6] Löfgren, K.G. (1979) The Corridor and Local Stability of the Effective Excess Demand Hypothesis: A Result. Scandinavian Journal of Economics, 81, 30-47. https://doi.org/10.2307/3439455

[7] Filippov, A.F. (1988) Differential Equations with Discontinuous Righthand Sides. Kluwer Academic Publishers, Dordrecht. https://doi.org/10.1007/978-94-015-7793-9

[8] Stiefenhofer, P. and Giesl, P. (2019) Economic Periodic Orbits: A Theory of Asymptotic Stability. Nonlinear Analysis and Differential Equations, 7, 9-16. https://doi.org/10.12988/nade.2019.923

[9] Borg, G. (1960) A Condition for the Existence of Orbitally Stable Solutions of Dynamical Systems. In: Kungliga Tekniska Högskolans Handlingar, Kungliga Tekniska Högskolan, Stockholm, Vol. 153, 3-12.

[10] Stiefenhofer, P. and Giesl, P. (2019) A Global Stability Theory of Nonsmooth Periodic Orbits: Example I. Applied Mathematical Sciences, 13, 511-520. https://doi.org/10.12988/ams.2019.9463

[11] Stiefenhofer, P. and Giesl, P. (2019) A Local Stability Theory of Nonsmooth Periodic Orbits: Example II. Applied Mathematical Sciences, 13, 521-531. https://doi.org/10.12988/ams.2019.9464 